\title{
Presentación de la menarca en madres e hijas. ¿Existe un adelanto?
}

\author{
Rubinstein $\mathrm{AV}^{1}$, Rahman $\mathrm{G}^{2}$, Risso $\mathrm{P}^{3}$, Ocampo $\mathrm{DC}^{4}$
}

\section{Resumen}

INTRODUCCIÓN: la menarca es un acontecimiento tardío en el desarrollo puberal y constituye el hito más significativo. Se ha descrito un adelanto progresivo en la edad de presentación de la menarca.

OBJETIVO: determinar la edad de aparición de la menarca y su relación con la edad de la menarca materna.

MATERIAL Y MÉTODOS: estudio retrospectivo, analítico, realizado entre mayo del 2014 y septiembre del 2015. Se estudiaron a niñas que presentaron su menarca con edad ginecológica menor a tres años. Se registró la edad de la menarca, seguro de salud y lugar de residencia de la niña y edad de la menarca de su madre, por el método de recuperación de datos. Se calcularon el índice de masa corporal (IMC) y el IMC/edad-Z score de la niña.

RESULTADOS: se incluyeron 250 niñas que presentaron menarca a una edad promedio de $11.6 \pm 1.1$ años; con un adelanto significativo de un año respecto a la de su madre: $12.6 \pm 1.6$ años $(p<0.00001)$. Se observó una correlación negativa estadísticamente significativa entre la edad de la menarca de las niñas y su IMC/edad-Z score $(p<0.00001)$; se mantuvo independiente del lugar de residencia $(p=0.3903)$ y del seguro de salud $(p=0.6067)$.

CONCLUSIÓN: en nuestra población, la menarca ocurrió un año antes en promedio en las hijas que en sus madres. Se observó una tendencia a la disminución de la menarca promedio en niñas con aumento del índice de masa corporal. No encontramos asociación entre la edad de la menarca y el nivel socioeconómico o lugar de residencia.

PALABRAS CLAVE: pubertad, menarca, madre, hija.

\section{Menarche in mothers and girls. Has age at menarche decline?}

Rubinstein $A V^{1}$, Rahman $G^{2}$, Risso $P^{3}$, Ocampo $D C^{4}$

\author{
Abstract \\ INTRODUCTION: Menarche is the most significant milestone in \\ female sexual maduration. It is a rather late event in puberty.
}

\begin{abstract}
${ }^{1}$ Jefa de la Unidad de Ginecología Infanto Juvenil. Especialista Consultor en Ginecología. Miembro de Comisión Directiva de la Sociedad Argentina de Ginecología Infanto Juvenil (SAGIJ). Certificación en Ginecología Infanto Juvenil (SAGIJ). Fellow en Ginecología Infanto Juvenil (FIGIJ). Hospital de Niños Sup. Sor María Ludovica de La Plata, Argentina.

${ }^{2}$ Médica de Planta de la Unidad de Ginecología Infanto Juvenil. Especialista Jerarquizada en Ginecología. Certificación en Ginecología Infanto Juvenil (SAGIJ). Fellow en Ginecología Infanto Juvenil (FIGIJ). Hospital de Niños Sup. Sor María Ludovica de La Plata, Argentina.

${ }^{3}$ Licenciada en Biología. Doctorando en Ciencias Médicas. Becaria en Comisión de Investigaciones Científicas. Docente de Cátedra de Bioestadística Bayesiana y Clásica (Facultad de Ciencias Veterinarias, UNLP) Comisión de Investigaciones Científicas (CIC). Cátedra de Bioestadística Bayesiana y Clásica. Facultad de Ciencias Veterinarias, Universidad Nacional de La Plata (UNLP).

${ }^{4}$ Médica de Planta de la Unidad de Ginecología Infanto Juvenil. Especialista Consultora en Pediatría. Certficación en Ginecología Infanto Juvenil (SAGIJ). Delegada de SAGIJ. Docente Cátedra de Pediatría A y Ginecología B (Facultad de Ciencias Médicas, UNLP) Hospital de Niños Sup. Sor María Ludovica de La Plata, Argentina.
\end{abstract}

Recibido: 13 de enero del 2016

Aceptado: 2 de diciembre del 2016

Correspondencia

Anahí Viviana Rubinstein

avrubinstein@yahoo.com.ar

Este artículo debe citarse como

Rubinstein AV, Rahman G, Risso P, Ocampo DC. Presentación de la menarca en madres e hijas. ¿Existe un adelanto? Acta Pediatr Mex. 2017;38(4):219-227. 
OBJECTIVE: To determine the menarcheal age and the relationship of menarcheal age between mothers and daughters.

MATERIAL AND METHODS: Retrospective, analytic study conducted between May 1st, 2014 and September 31st, 2015. Girls who had menarche with gynecological age less than three years were analyzed . The age of menarche of the girl and her mother, health insurance and place of residence was recorded by the method of retrieving data. BMI and $\mathrm{BMI} / \mathrm{age}-\mathrm{Z}$ score were calculated.

RESULTS: Two hundred and fifty girls were included. The mean age at menarche was 11.6 years. The mean menarcheal age of the mothers (12.6 years) was higher than the mean menarcheal age of the girls. Statistically significant difference was observed between the menarcheal age of the girls and mothers $(p<0.00001)$. A statistically significant negative correlation between age of menarche of girls and $\mathrm{BMI} /$ age$Z$ score $(p<0.00001)$ was observed and remained independent of the place of residence $(p=0.3903)$ and health insurance $(p=0.6067)$.

CONCLUSIONS: Menarche occurs earlier in the daughters than in their mothers in our population. A trend to decreased average menarche in girls with increased BMI was observed. The age of menarche of girls remained independent of their socioeconomic status or place of residence.

KEYWORDS: puberty; menarche; mother; girl
Correspondence

Anahí Viviana Rubinstein avrubinstein@yahoo.com.ar

\section{INTRODUCCIÓN}

La pubertad se caracteriza por una serie de cambios físicos y psicológicos a lo largo de un período aproximado de tres o cuatro años, inducidos por un aumento paulatino de esteroides sexuales. ${ }^{1}$ En esta etapa, se registra la interacción dinámica entre factores genéticos y ambientales. ${ }^{2}$

La menarca es el hito más significativo en el desarrollo puberal femenino. Ocurre dos años después de la aparición de la telarca y con estadios de Tanner mamario III-IV, luego de la fase máxima de velocidad de crecimiento. Es un acontecimiento tardío en la cronología puberal. Varios estudios centraron su interés en la menarca como un indicador de las al- teraciones del desarrollo puberal. La edad de la menarca se acepta como un rasgo fiable para evaluar y comparar poblaciones, ya sea en un momento y situación específica, como a lo largo de un período; es un hecho fácil de recordar y registrar. ${ }^{3}$

Para las niñas estadounidenses el promedio de edad de la menarca correspondió a 12.8 años. ${ }^{4}$ Los estudios NHANES (National Health and Nutrition Examination Survey) establecieron una disminución de la edad de la menarca de 13.2 a 12.2 años en niñas mexicanas-americanas; de 13.3 a 12.5 en niñas blancas no hispanas y de 13.6 a 12.2 en niñas negras no hispanas. ${ }^{5}$ En Cuba, las niñas que residían en las zonas urbanas tuvieron la menarca antes que las que residían 
en zonas rurales, 12.6 vs.13 años. ${ }^{6}$ En Chile, en una población escolarizada de 1,302 niñas, la edad de la menarca correspondió a 12.7 años. ${ }^{7}$ En Argentina, estudios publicados por Lejarraga y Méndez-Ribas determinaron la edad de menarca en 12.6 años. ${ }^{8,9}$

Estudios epidemiológicos evidencian que la menarca se ha ido adelantando progresivamente desde mediados del siglo XIX; hecho conocido como tendencia secular de la menarca. La reducción de la edad de la menarca constituye el marcador más fiable de los cambios seculares positivos en el desarrollo puberal..$^{2,10}$

\section{OBJETIVO}

Determinar la edad de aparición de la menarca y su relación con la edad de la menarca materna en una población hospitalaria. Caracterizar a las adolescentes según su lugar de residencia, seguro de salud e índice de masa corporal (IMC) y relacionar dichas variables con la edad de la menarca.

\section{MATERIAL Y MÉTODOS}

Estudio observacional, retrospectivo, transversal, analítico. Entre mayo 2014 y septiembre 2015, se revisaron las historias clínicas de niñas que presentaron su menarca con edad ginecológica menor a tres años, evaluadas en la Unidad de Ginecología Infanto-Juvenil del Hospital de Niños Superiora Sor María Ludovica de la ciudad de La Plata, Argentina, cuyo motivo de consulta fue examen de salud de la adolescente o afecciones especificas ginecológicas. La atención es estatal y gratuita, asiste a la población de la provincia de Buenos Aires.

Se registró la edad de la menarca, lugar de residencia y seguro de salud por el método retrospectivo o de recuperación de datos. Se caracterizó a las pacientes según el lugar de resi- dencia en tres grupos: urbana, suburbana y rural; y según la presencia o ausencia de seguro de salud como un indicador del nivel socioeconómico que representa la formalidad del trabajo. ${ }^{11} \mathrm{Se}$ entrevistó a las pacientes y al acompañante luego de haber acontecido la menarca en las niñas. El mismo método se utilizó para la menarca materna. En las niñas se obtuvo el peso, determinado mediante balanza convencional con precisión de 100 g y la talla obtenida con estadiómetro de pared convencional. Se calculó el índice de masa corporal (IMC kg/cm²); los resultados se presentan como desviación estándar (DE) para la edad (puntaje Z). ${ }^{12}$

\section{Criterios de exclusión}

Se excluyeron las pacientes en quienes no se obtuvo el registro de la edad de la menarca materna, casos de adopción y presencia de patologías crónicas.

\section{Análisis estadístico}

Se estimó el tamaño de la muestra para la comparación de dos medias ${ }^{13}$ (Cuadro 1). El tamaño de la muestra calculado fue de 137 niñas y 137 madres para mostrar si hubo una diferencia de al menos 6 meses en el registro de la edad promedio de la menarca. La edad de la menarca fue analizada en función de las variables cualitativas nominales, lugar de residencia y seguro de salud. En ambos casos se utilizó ANOVA como método estadístico.

\section{Cuadro 1. Estimación del tamaño de la muestra}

Tamaño de muestra $=\left(2 *(Z \alpha+Z \beta)^{2} * S^{2}\right) / d^{2}=137$

Fueron considerados:

- Riesgo de 0.05 (Z $\alpha:$ 1.96)

- Poder estadístico de 95\% (Zß: 1,645)

- Varianza de la edad de la menarca en las niñas del presente estudio $\left(\mathrm{S}^{2}: 1.31\right.$ años)

- Diferencia en la edad de menarca respecto a las madres, de al menos 6 meses (d: 0.5 años) 
El valor de IMC/edad-Z score fue calculado con el software WHO Anthro Plus. ${ }^{12}$ Se analizó cuantitativamente y como variable categórica, considerando cinco grupos: desnutrición $(Z<-2)$, bajo peso ( $Z$ entre -1 y -2 ), peso normal ( $Z$ entre -1 y 1$)$, sobrepeso $(Z>1 \leq 2)$ y obesidad $(Z>2){ }^{12,14,15}$ Debido a la gran variación del $Z$ score en la población se procedió al cálculo de su mediana. Asimismo, la relación entre la edad de la menarca de las niñas y el Z score fue evaluada a través del coficiente de correlación de Spearman (R).

Las pacientes fueron categorizadas en dos grupos según el motivo de consulta: sintomático (trastorno del ciclo menstrual, patología de ovario y dolor abdominal) y asintomático (patología de la mama, de la vulva, asesoramiento en anticoncepción y examen de salud del paciente). La edad de la menarca fue comparada entre estos grupos. Se comparó la edad de la menarca entre madres y niñas como relación simple y en relación a las categorías sintomático/asintomático y según las categorías de Z score. Estos análisis se realizaron mediante ANOVA. La prueba de Levene se utilizó para probar la homogeneidad de varianzas. El nivel de significancia elegido fue $p<0.05$. Se utilizó el programa Microsoft Excel ${ }^{\circledR}$ como base de datos e InfoStat versión 2014 para el análisis estadístico. ${ }^{16}$

\section{Consideraciones éticas}

Debido al carácter retrospectivo del estudio no se requiere consentimiento informado. El estudio fue aprobado por la Sala de Docencia e Investigación del Hospital Superiora Sor María Ludovica.

\section{Consideraciones para evitar sesgos potenciales}

Debido a que la edad de la menarca fue una pregunta central en la entrevista, en el caso de las niñas, sólo fueron considerados aquellos registros en los que la edad de la menarca fuese precisa por la cercanía en el tiempo con el evento (edad ginecológica $<3$ años). Se unificaron los criterios y los instrumentos de evaluación entre los profesionales intervinientes. La edad de la menarca en madres e hijas fue evaluada según motivo de consulta (con/sin sintomatología) y categorías de Z score, transformando estas variables confusoras en variables control.

\section{RESULTADOS}

\section{Población de estudio}

Durante el período de mayo del 2014 a septiembre del 2015 fueron atendidas 273 niñas. Se excluyeron 23: 5 no presentaron registro de la menarca materna al momento de la evaluación; 3 eran adoptadas y 15 presentaban afecciones crónicas (leucemias: 4; hipotiroidismo: 4; pubertad precoz: 2 ; insuficiencia renal crónica: 2 ; enfermedad fibroquística del páncreas: 2; osteosarcoma: 1). La edad promedio de las 250 niñas en estudio fue 12.9 años (DE: 1.4) con un intervalo entre 9.8 y 16 años. La edad de la menarca promedio de las niñas fue de 11.6 años (DE: 1.1).

Se dividieron a las pacientes de acuerdo con el motivo de consulta en asintomáticas: examen de salud de la adolescente n: $83 ; 33.2 \%$; asesoramiento en anticoncepción n: 15; 6.0\%; patología de la mama n: $6 ; 2.4 \%$ y patología de la vulva (n: $18 ; 7.2 \%)$. Y sintomáticas: trastornos del ciclo menstrual n: $98 ; 39.2 \%$; dolor abdominal n: $15 ; 6.0 \%$ y patología del ovario n: 15 ; $6.0 \%$. Se registraron 128 niñas sintomáticas y 122 asintomáticas. La edad de la menarca fue 11.61 (DE: 1.14) y 11.56 (DE: 1.16), respectivamente, sin encontrar diferencia estadísticamente significativa entre las pacientes sintomáticas y asintomáticas $(p=0.7244)$.

Las pacientes sin seguro de salud fueron $77.2 \%$ (n: 193). El lugar de residencia de las niñas correspondió en $68.4 \%$ (n: 171) a zonas urbanas; 
$26.4 \%$ (n: 66) a zonas suburbanas y $5.2 \%$ (n: 13 ) a zona rural. No se encontraron diferencias estadísticamente significativas en la edad de la menarca entre las niñas con y sin seguro de salud $(p=0.6067)$, ni entre las niñas que habitaron zonas urbanas, suburbanas y rurales $(p=0.3903)$. Para descartar un posible efecto del bajo porcentaje de niñas que viven en zonas rurales, se analizó nuevamente excluyendo este grupo y se observó la misma tendencia $(p=0.5661)$.

La mediana de IMC/edad-Z score fue 1, oscilando entre -2.47 y 4.02 . Se registraron 119 niñas (47.6\%) con peso normal, 78 niñas (31.2\%) con sobrepeso y 45 niñas (18\%) obesas, 6 niñas $(2.4 \%)$ con bajo peso y 2 niñas $(0.8 \%)$ con desnutrición. Se observó una correlación negativa estadísticamente significativa entre la edad de la menarca de las niñas y su IMC/edad-Z score $(\mathrm{R}=-0.28 ; p<0.00001)$ (Figura 1).

\section{Menarca entre madres e hijas}

Se encontró que la edad de la menarca difirió significativamente entre madres e hijas

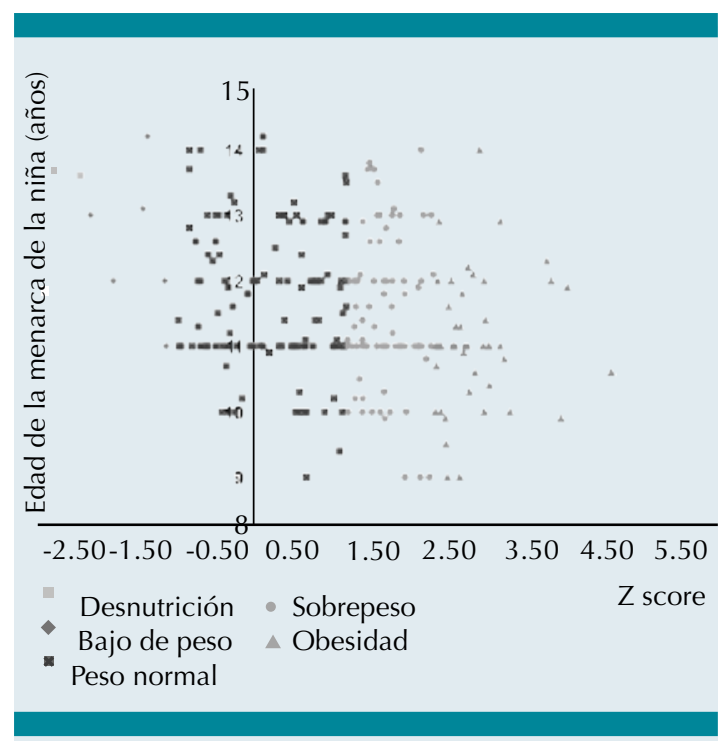

Figura 1. Relación entre la edad de la menarca de la niña y el índice de masa corporal/edad-Z score. $(p<0.00001)$. La edad promedio de estas últimas fue de 11.6 años (DE: 1.1) y la de sus madres: 12.6 años (DE: 1.6). Se registró un adelanto de un año en promedio. Se observó que la edad materna presentó mayores oscilaciones, con un rango de edad que duplicó el valor hallado en las niñas (Cuadro 2). La Figura 2 muestra la dinámica de la edad de la menarca en ambos grupos. En relación con la edad de la menarca entre madres e hijas según el motivo de consulta de las niñas (sintomáticas o no sintomáticas) se encontraron

Cuadro 2. Comparación de la edad de la menarca (en años) de madres e hijas

\begin{tabular}{|l|c|c|c|c|}
\hline Grupo & Media (DE) & Intervalo & Rango & CV\% \\
\hline Hijas (n: 250) & $11.6(1.1)$ & $9.0-14.2$ & 5.2 & 9.9 \\
\hline Madres (n: 250) & $12.6(1.6)$ & $8.0-18.0$ & 10.0 & 12.8 \\
\hline
\end{tabular}

CV\%: coeficiente de variación porcentual.

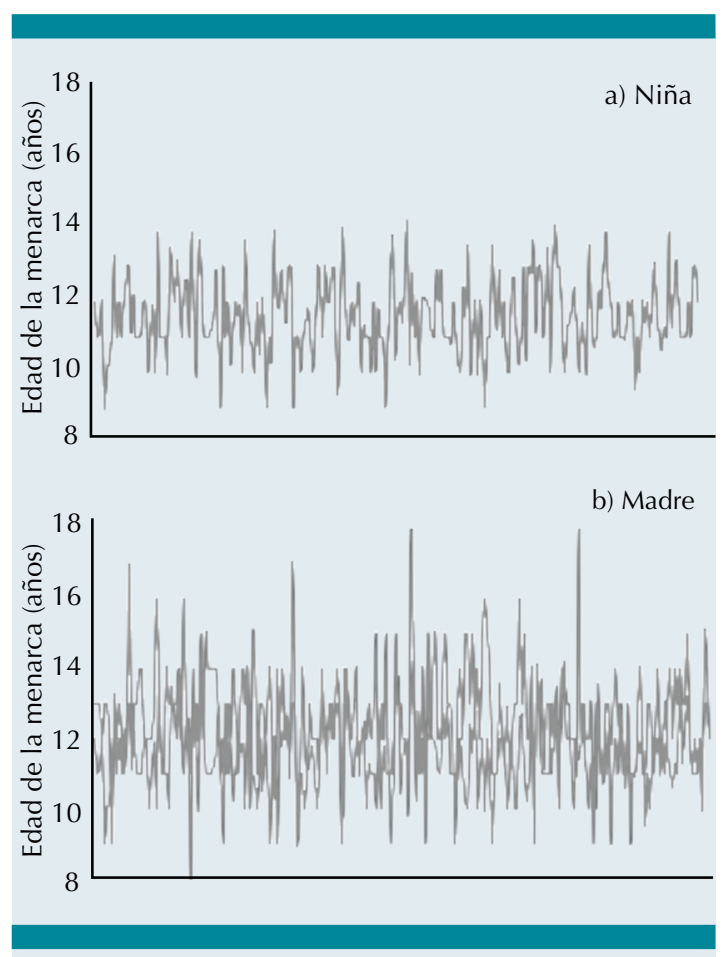

Figura 2. Edad de la menarca de la niña (inciso a) y su madre (inciso b). 
diferencias significativas $(p<0.00001)$. La menarca de las niñas respecto a la de sus madres se adelantó en promedio en 12.5 meses en las niñas sintomáticas y 11.2 meses en las niñas asintomáticas (Cuadro 3).

Al analizar esta relación de acuerdo con el Z score, se observó una tendencia a menor edad promedio de la menarca en niñas con sobrepeso $(\mathrm{F}=20.83 ; p<0.0001)$ u obesidad $(\mathrm{F}=21.26$; $p<0.0001)$, manteniendo un adelanto estadísticamente significativo en la edad de la menarca en las niñas en comparación con sus madres (Cuadro 4). En las niñas con desnutrición o bajo peso se observó un retraso de la edad de la menarca con respecto a la de sus madres, pues al ser un grupo reducido no se pudieron demostrar diferencias estadísticamente significativas ( $p=0.159$ y $p=0.641$, respectivamente).

\section{DISCUSIÓN}

Uno de los hechos más significativos en la cronología puberal es su adelanto progresivo. De 1830 a 1860 la edad de la menarca disminuyó en 2 a 3 meses por década, declinando de 17 a 13 años durante ese período. ${ }^{17}$ De 1970 al 2000 la edad de la menarca disminuyó de 12.8 a 12.6 en niñas blancas, a 12.1 en niñas negras y a 12.2 años en sudamericanas. ${ }^{18-20}$ Tehrani y sus colaboradores describieron la disminución de la edad media de la menarca de 13.8 a 12.9 años entre 1930 y $1990 .{ }^{21}$ Sin embargo, no hay consenso si a partir de 1970 la edad de la menarca ha continuado adelantándose o si se ha detenido..22 En la población chilena la edad promedio es semejante a la observada en los países europeos, con un adelanto progresivo hasta 1970 y estabilización posterior. ${ }^{23}$ Papadimitriou y su grupo no reportaron diferencias significativas en la edad de la menarca en niñas residentes en Atenas entre 1996-2006: 12.3 años. ${ }^{24}$ La Sociedad Argentina de Ginecología Infanto Juvenil registró la edad media de la menarca en 12.2 años. ${ }^{25}$ En la población hospitalaria evaluada en este trabajo la media fue de 11.6 años.

Se publicó que la menarca de las hijas ocurre en época más temprana que la de su madre. Méndez Ribas y sus colegas registraron la edad de la menarca en 12.6 en las niñas y la edad de la menarca materna en 13.4 años. ${ }^{9}$ Para Guillén y sus colaboradores la media de edad de la menarca en las adolescentes fue de 12.2 años y la

Cuadro 3. Edad de la menarca en niñas y madres según motivos de consulta (sintomático/asintomático)

\begin{tabular}{|c|c|c|c|c|c|c|c|}
\hline Categoría & Grupo & Casos & Media & DE & Intervalo & Razón-F & Valor-p \\
\hline \multirow{2}{*}{ Sintomático } & Niña & 128 & 11.61 & 1.14 & $9-14.2$ & \multirow{2}{*}{32.21} & \multirow{2}{*}{$<0.00001$} \\
\hline & Madre & 128 & 12.65 & 1.74 & $8-18$ & & \\
\hline \multirow{2}{*}{ Asintomático } & Niña & 122 & 11.56 & 1.16 & $9-14.2$ & \multirow{2}{*}{30.13} & \multirow{2}{*}{$<0.00001$} \\
\hline & Madre & 122 & 12.49 & 1.47 & $9-16$ & & \\
\hline
\end{tabular}

Cuadro 4. Edad de la menarca en niñas y madres en cinco categorías de IMC/edad- Z score

\begin{tabular}{l|cc|cc|cc|cc|cc}
\hline Z score & \multicolumn{3}{|c|}{ Desnutrición (2) } & \multicolumn{2}{c|}{ Bajo peso (6) } & \multicolumn{2}{c|}{ Peso normal (119) } & \multicolumn{2}{c|}{ Sobrepeso (78) } & Obesidad (45) \\
\hline Grupo & Media & DE & Media & DE & Media & DE & Media & DE & Media & DE \\
Niñas & 13.6 & 0.00 & 12.55 & 1.12 & 11.74 & 1.12 & 11.5 & 1.14 & 11.07 & 1.01 \\
\hline Madres & 12.5 & 0.71 & 12.17 & 1.6 & 12.66 & 1.56 & 12.55 & 1.69 & 12.42 & 1.69
\end{tabular}

IMC: índice de masa corporal. 
de sus madres fue de 12.7. ${ }^{26}$ En Turquía, Ersoy y su grupo estudiaron 1,017 niñas escolarizadas, cuya edad de la menarca correspondió a 12.8 y la edad de la menarca materna a 13.6 años. Esta relación se mantuvo independiente de la situación socio-económica, el estado nutricional y la actividad física de las niñas. ${ }^{27}$

En nuestro estudio la edad promedio de la menarca en las niñas fue 11.6 vs. 12.6 años en las madres. La edad de la menarca varió significativamente entre la madre y la hija con un año de adelanto. Si bien el determinante principal del desarrollo cronológico de la pubertad es genético o hereditario, la aparición de la menarca no puede atribuirse a un solo factor. Existen distintos factores que influyen en el momento del inicio y la velocidad de progresión de la pubertad: localización geográfica, exposición a la luz, a disruptores endócrinos, nutrición, obesidad, así como factores psicológicos. ${ }^{4,10}$ La tendencia secular sigue siendo observada en los países en desarrollo debido a los cambios actuales en la calidad de vida. En recientes reportes en Nigeria, Guatemala y Colombia la edad de la menarca es más tardía que en Chile y Venezuela. ${ }^{28,29}$ Es posible que las condiciones de vida puedan haber alcanzado un nivel óptimo en la expresión del potencial genético.

En países en desarrollo, las inequidades relacionadas al status económico o al lugar de residencia, urbano-rural, siguen siendo relevantes y pueden dar cuenta de importantes variaciones en la pubertad..$^{30}$ En nuestro trabajo, la edad de la menarca en las niñas evaluadas se mantuvo independiente del lugar de residencia y seguro de salud. En relación con los diferentes motivos de consulta no hubo diferencias significativas entre pacientes sintomáticas y asintomáticas. Por lo tanto, el antecedente de alteraciones del ciclo menstrual, patología ovárica y dolor abdominal no parecerían influir en la edad de aparición de la menarca. Las pacientes con menarca temprana muestran un riesgo incrementado de obesidad, signos clínicos de resistencia a la insulina y patología cardiovascular. ${ }^{31}$ Hernández y sus colegas demostraron que las niñas que presentaron la menarca antes de los 11.5 años tuvieron mayor índice de masa corporal y mayor sobrepeso u obesidad. ${ }^{7}$ En coincidencia, en nuestro estudio, se observó una disminución de la edad de la menarca promedio en niñas con mayor índice de masa corporal. Una explicación posible de estos hallazgos podría ser la adiposidad como factor determinante en la aceleración de la maduración biológica.

Según Hamilton y sus colaboradores existe riesgo de cáncer de mama en mujeres con menarca temprana y con antecedentes familiares de esta patología. ${ }^{32}$ En nuestro trabajo no hubo registro alguno de cáncer de mama en las madres de las pacientes. Sería necesario ampliar las investigaciones en este campo a fin de orientar conductas preventivas en patologías prevalentes.

Los análisis de tendencia secular a menudo están limitados por la comparación entre las investigaciones que incluyen diferentes poblaciones y períodos de tiempo. En nuestro estudio, el tamaño de muestra requerido para demostrar al menos 6 meses de adelantamiento/retraso en la edad de la menarca entre madres e hijas fue superado (137 vs. 250 registros). No obstante, la cantidad de niñas desnutridas y con bajo peso según su Z score fueron escasas ( 2 y 6 casos, respectivamente). Para tener una mayor validez externa de los resultados los cinco grupos de Z score deberían estar distribuidos más equitativamente. Existen pocos estudios en nuestro país que hagan referencia al adelanto de la edad de presentación de la menarca de las niñas en relación a la de sus madres.

\section{CONCLUSIÓN}

La menarca ocurrió un año antes en las hijas que en sus madres, en promedio, demostrando un 
adelanto de la presentación de la menarca en la población estudiada. Se observó una tendencia a disminución de la edad promedio de la menarca en niñas con Z score del índice de masa corporal mayor de 1. No encontramos asociación entre la edad de la menarca y el nivel socioeconómico o el lugar de residencia.

\section{REFERENCIAS}

1. Azaretzky $\mathrm{M}$, Boquete $\mathrm{H}$, Llano $\mathrm{M}$, y col. Cap. 3.7 Pubertad normal. En Sociedad Argentina de Ginecología Infanto Juvenil. Ginecología Infanto juvenil Un abordaje interdisciplinario. Ediciones Journal 2015. Buenos Aires;137-143.

2. Karapanou O, Papadimitriou A. Determinants of menarche. Reprod Biol Endocrinol. 2010;8:115. doi: 10.1186/14777827-8-115

3. Prado $C$, Jiménez JM, Rubén $M$. La edad de la menarquía y aparición de caracteres sexuales secundarios; cambio secular en la población cubana (1972-2000). Rev Esp Antropo Biol. 2002;23:57-64.

4. Speroff L, Fritz M. Cap. 10 Anomalías de la pubertad y problemas del crecimiento en: Endocrinología Ginecológica Clínica y Esterilidad 70 Edición 2007 Editorial Lippincott Williams Wilkins. pp.361-399.

5. McDowell M, Brody D and Hughes J. Has Age at Menarche Changed? Results from the National Health and Nutrition Examination Survey (NHANES) 1999-2004. Journal of Adolescent Health. 2007;40: 227-231.

6. Valdés Gómez W, Espinosa Reyes T, Leyva Álvarez de la Campa G. Menarquía y factores asociados. Revista Cubana de Endocrinología. 2013;24(1):35-46.

7. Hernández $\mathrm{M}$, Unanue N, Gaete X y col. Edad de la menarquía y su relación con el nivel socioeconómico e índice de masa corporal. Rev Méd Chile. 2007;135:1429-36.

8. Lejarraga H. Age of menarche in urban Argentinian girls. Ann Hum Biol. 1980;7:579-581.

9. Méndez JM, Siemazko K. Trastornos del ciclo menstrual durante el período puberal. En: Enfoque actual de la adolescente por el ginecólogo. Buenos Aires. Editorial Ascune, 2005. p.138-156.

10. Lalys L, Pineau JC. Age at menarche in a group of French schoolgirls. Pediatrics International. 2014;56:601-604.

11. Azcárate, P. y Zambelli, R. El nivel socioeconómico en la argentina, 2015. Estratificación y variables. Observatorio Social de SAIMO. 1 de Julio 2015. Recuperado el 23/2/ 2016 de: http://www.saimo.org.ar/archivos/observatoriosocial/EI-NSE-en-la-Argentina-2015-Estratificacion-yVariables.pdf

12. WHO AnthroPlus for personal computers Manual:Software for assessing growth of the world's children and ado- lescents. Geneva: WHO, 2009 (http://www.who.int/ growthref/tools/en/).

13. Basualdo-Farjat, J, Grenóvero, S, Minvielle, M. Nociones básicas de metodología de investigación en ciencias de la salud. Tomo 2. Gráfica Alemana. Editorial Atlante. 2004.

14. World Health Organization. Training course on child growth assessment. WHO, Geneva 2008. Copyright $(2008$ World Health Organization. http://www.who.int/childgrowth/ training/module_c_interpreting_indicators.pdf

15. de Onis M, Onyango AW, Borghi, Siyam A, et al. Developmen of a WHO growth reference for school-aged children and adolescents. Bull World Health Organ 2007;85(9):660-7.

16. Di Rienzo JA, Casanoves F, Balzarini MG, Gonzalez L, Tablada M, Robledo CW. Infostat versión 2014. Group Infostat, FCA, Universidad Nacional de Córdoba, Agentina. Disponible en http://infostat.com.ar (acceso 09/10/2015).

17. Tanner JM, Eveleth PB Variability between populations in growth and development at puberty In: Berenberg SR (ed). Puberty. Biologyc and Psychosocial Components. Stenfert Kroese, Leiden, the Netherlands, 1975:256-273.

18. Kaplowitz P. Pubertal development in girls: Secular trends. Curr.Opin.Obstet. Gynecol. 2006;18: 487-491.

19. Herman-Giddens ME,Slora EJ, Wasserman RC et al Secondary sexual characteristics and menses in young girls seen in office practice: A study from the pediatrics research in office settings. Pediatrics. 1997;99:505-512.

20. Herman-Giddens ME Recent data on pubertal milestones in United States children: The secular trend toward earlier development. Int. j. Androl. 2006;29:241-246.

21. Tehrani F.R, Mirmiran P, Zahedi S: Menarcheal age of mothers and daughters: Tehran Lipid and Glucose Study. Eastern Mediterranean Health Journal EMHJ. 2010;16(4):391-395.

22. Parent, AS; Teilmann, G; Juul A; Skakkebaek, NE; Toppari, $\mathrm{J}$; Bourguignon, JP The timing of normal puberty and the age limits of sexual precocity: Variations around the world, secular trends, and changes after migration. Endocrine Reviews. 2003;24(5):668-693.

23. Gaete X, Codner E. Adelanto de la pubertad en Chile y el mundo. Rev Chil Pediatr. 2006;77:5:456-465.

24. Papadimitriou A, Fytanidis G, Douros K: Age at menarche in contemporary Greek girls: evidence for levelling-off of the secular. Act.Pediatrica. 2008;97:812-815.

25. Salvo, ME; Daldevich, D; Vázquez, S; Paccetti, J; Varela, S. Investigación exploratoria sobre salud sexual y reproductiva y prevalencia de violencia familiar y de pareja en la adolescencia. Rev Soc Arg Ginecol. 2011;18:2:80-91.

26. Guillén L, Blanco Rodríguez, Martín M, Martínez Carmela. Secular trend of menarcheal age in Spanish adolescents Medicina Clínica. 2008;131(9):355-356.

27. Ersoy B, Balkan C, Gunay $T$ and Egemens A: The factors affecting the relation between the menarcheal age of mother and daughter. Child: Care, Health \& Development. 2005;31(3):303-308. 
28. Macias-Tomei C, Lopez-Blanco M, Espinoza I, VasquezRamirez M. Pubertal development in Caracas uppermiddle-class boys and girls in a longitudinal context. Am J Hum Biol. 2000;12:88-96.

29. Carrillo JC, Ireton MJ, Caro LE, Hauspie R, Morales JC, Pagezy H. Growth Traits an sexual and sexual maturation in a simple of school-children from El Yopal, Casanare, Colombia. Acta Med Auxol. 2001;33:105-111.

30. Eveleth PB, Tanner JM. Cap 8.Sexual development. In Eveleth PB,ed. Worldwide variation in human growth.
Cambridge, England: Cambridge University Press.1990. 2nd Ed;161-175.

31. Remsberg KE, Demerath EW, Schubert CM: Early menarche and the development of cardiovascular disease risk factors in adolescent girls: the Fels Longitudinal Study. I Clin Endocrinol Metab. 2005;90:2718-2724.

32. Hamilton AS, Mack TM: Puberty and genetic susceptibility to breast cancer in a case-control study in twins. N Engl J Med. 2003;348:2313-2322. 